\title{
2. \\ Continuous Chemical Synthesis in Flow
}

\author{
Ian R. Baxendale \\ Departrment of Chemistry, University of Durham, South Road, Durham. DH1 3LE, UK. \\ e-mail: i.r.baxendale@durham.ac.uk
}

Keywords: Flow chemistry, Synthesis, Heterocycles

\section{Abstract Speech}

Synthetic organic chemistry is a complex art, science, craft and to many a pure religion. As a scientific discipline it continues to undergone considerable change and evolution. Indeed, over the past couple of centuries our understanding of the fundamentals methodologies applied to bond forming and breaking including how to exert control over these processes has progressed at a stunning pace. It is probably fair to say synthetic chemists can sufficient time could prepare any required molecule imaginable. However, throughout this constant change the basic approach and apparatus available to the bench chemist has remained relatively constant. The prevalence of test tubes, round bottom flasks and beakers are historically synonymous with chemistry laboratories. This image remains essential identical today in that our approach to synthesis is mainly still a batch based practice following well defined iterative sequence of discrete reaction steps, work-ups and purifications using historically recognizable glassware.

An alternative approach currently being explored is flow chemical processing which makes use of small volume reactors and packed bed columns as dynamic vessels for performing chemical transformations. ${ }^{1}$ This more holistic approach also attempts to integrate other aspects of the synthesis such as work-up and purification into the overall processing sequence aiming to deliver a clean product stream as one single integrated operation. Consequently the ability to further telescope and assimilate these individual transformations into more elaborate sequences enables the generation of advanced multi-step product outputs.

This presentation will detail some of the achievements from our laboratory in the area of flow chemistry with emphasis being placed on the application of these techniques to synthetic organic chemistry. By way of exemplification a selection of small molecule building blocks and pharmaceutical libraries will be illustrated.

\section{REFERENCES}

1 (a) I. R. Baxendale, C. J. Millia, L. Brocken, Green Process Synth. 2013 2, 211-230. (b) I. R. Baxendale, J. Chem. Technol. Biotechnol. 2013, 88 $519-552$. 